\title{
Runt-related Transcription Factor 1 (RUNX1T1) Suppresses Colorectal Cancer Cells Through Regulation of Cell Proliferation and Chemotherapeutic Drug Resistance
}

\author{
MUSAAD ALFAYEZ, RADHAKRISHNAN VISHNUBALAJI and NEHAD M. ALAJEZ \\ Stem Cell Unit, Department of Anatomy, College of Medicine, \\ King Saud University, Riyadh, Kingdom of Saudi Arabia
}

\begin{abstract}
Background/Aim: Altered expression of runt-related transcription factor 1 (RUNX1T1) has been observed in several human cancer types; however, the exact role for RUNX1T1 in colorectal cancer $(C R C)$ remains unclear. Materials and Methods: The GSE21510 CRC and our previously published datasets were utilized in this study. Gene-expression profiling was conducted using the Agilent microarray platform, while data normalization and bioinformatics were conducted using GeneSpring software. AlamarBlue assay was used to assess cell viability in vitro. Results: The expression of RUNXIT1 was severely down-regulated in primary $C R C$ and cell lines. Lentiviral-mediated re-expression of RUNX1T1 inhibited CRC cell growth, and global gene-expression analysis revealed the cell cycle, DNA replication, and DNA damage as the pathways most affected by RUNX1T1. Forced expression of RUNXIT1 induced a significant reduction in cellular proliferation and sensitized CRC cells to 5-flurouracil. Conclusion: Our data revealed a novel role for RUNXIT1 as a tumor-suppressor gene in CRC through modulation of multiple cellular pathways.

Colorectal cancer (CRC) is among the most prevalent types of cancer worldwide, thus causing a major health burden due to its high mortality rates (1). Advances in our understanding of the diagnostic, prognostic and predictive value of novel molecular markers could potentially have a significant impact on the clinical management of this disease. In particular, identifying novel molecular mechanisms that drive disease progression or therapy resistance is very crucial for future management of this disease.
\end{abstract}

Correspondence to: Musaad Alfayez, Stem Cell Unit, Department of Anatomy, College of Medicine, King Saud University, Riyadh 11461, Kingdom of Saudi Arabia. Tel: +966 14679421, Fax: +966 14671498,e-mail: alfayez@ksu.edu.sa

Key Words: RUNX1T1, colorectal cancer, gene expression, chemoresistance.
Previous studies of CRC have identified key genes driving CRC development and progression. Our recent work revealed multiple deregulated genes in CRC (2). Therefore, it is generally believed that CRC evolves through multiple alterations due to genetic or epigenetic mechanisms.

Runt-related transcription factor 1 (RUNX1T1) is a transcriptional co-repressor and an important regulator of leukemogenesis. RUNX1T1 gene encodes a member of myeloid translocation genes, which interact with DNAbound transcription factors and recruit a range of corepressors to facilitate transcriptional repression (http://www.ncbi.nlm.nih.gov/gene/862) (3-5).

Mutation rates and the sets of mutated genes vary considerably across tumor types (breast, lung, ovarian and prostate cancer) and subtypes. Statistical analysis revealed 77 significantly mutated genes, including RUNX1T1 (6). Genome-wide mutational screening revealed that RUNX1T1 was somatically mutated in a number of solid human cancer types; however, point mutations of RUNX1T1 are largely unknown $(6,7)$. Furthermore, the genomic landscape was analyzed in CRC and identified RUNX1T1 somatic point mutations (8).

In this study, we sought to determine the role of RUNX1T1 in CRC progression thorough investigating its expression in a set of CRC mRNA datasets combined with bioinformatics and gain-of-function approaches.

\section{Materials and Methods}

Cells lines and tissue culture. The HCT116 human colorectal cancer cell line was obtained and subsequently was authenticated by Genetica DNA Laboratories, Inc. (Burlington, NC, USA). Cells were maintained in Dulbecco's modified Eagle's medium (DMEM) supplemented with $10 \%$ fetal bovine serum (Gibco-Invitrogen, Waltham, MA, USA) and $100 \mathrm{mg} / 1 \mathrm{penicillin} / \mathrm{streptomycin}$. All cells were maintained in an incubator at $37^{\circ} \mathrm{C}$ in a humidified atmosphere with $5 \% \mathrm{CO}_{2}$.

Lentiviral transduction. Transduction was peroformed in accordance with our published protocols (9). Briefly, lentiviral particles encoding for RUNX1T1 or control lentiviral particles were 
purchased from Genecopoeia Inc., (Rockville, MD, USA). Two hundred thousand HCT116 cells were seeded in complete DMEM in 24-well plate. Twenty-four hours later ( $~ 80$ confluency), the medium was removed and then $20 \mu \mathrm{l}$ of crude lentiviral particles in $500 \mu \mathrm{l}$ of DMEM with $5 \%$ heat-inactivated serum (Invitrogen) and $1 \%$ Pen-Strep supplemented with polybrene $(8 \mu \mathrm{g} / \mathrm{ml}$; Sigma, St. Louis, MO, USA) was added to the cells. Seventy-two hours later, the medium was removed and transduced cells were selected with puromycin $(1 \mu \mathrm{g} / \mathrm{ml}$; Sigma) for 1 week until stably transduced cells were generated.

Gene-expression microarray. RNA isolation, gene and microRNA expression experiments were performed in accordance with our published protocols $(9,10)$. In brief, RNA was isolated using Total Tissue RNA Purification Kit from Norgen-Biotek Corp. (Thorold, ON, Canada) and were quantified using NanoDrop 2000 (Thermo Scientific, Wilmington, DE, USA). Total RNA was labelled and then hybridized to the Agilent Human SurePrint G3 Human GE 8×60 k mRNA microarray chip (Agilent Technologies, Palo Alto, CA, USA). All microarray experiments were conducted at the Microarray Core Facility (Stem Cell Unit, Department of Anatomy, College of Medicine, King Saud University). Data were subsequently normalized and analyzed using GeneSpring 13.0 software (Agilent Technologies). Pathway analyses were conducted using the Single Experiment Pathway analysis feature in GeneSpring 13.0 (Agilent Technologies). A two-fold cut-off with $p<0.02$ was used.

Measurement of cell viability. The viability of lentiviral control and RUNX1T1-transduced HCT116 cells was determined using AlamarBlue assay as described elsewhere (10). All assays were carried out with appropriate controls. Briefly, 5,000 cells were cultured in a 96-well plate and cell viability was measured at different time points by adding AlamarBlue assay reagent $(10 \%$ by volume) and measuring absorbance at $570 \lambda$.

Measurement of apoptosis. Fluorescence-based apoptosis was determined in cells after exposure to different concentrations (1.5$24 \mu \mathrm{M}$ ) of 5-flurouracil (5-FU; EBEWE Arznetmittel Ges.m.b.H., Austria), using acridine orange (AO) and ethidium bromide (EB) staining method. The differential uptake of AO/EB allows the identification of viable and non-viable cells. AO was used to visualize the number of cells which had undergone apoptosis. After treatment, the lentiviral control and RUNX1T1-transduced HCT116 cells were stained with dual fluorescent staining solution containing $100 \mu \mathrm{g} / \mathrm{ml} \mathrm{AO}$ and $100 \mu \mathrm{g} / \mathrm{ml}$ EB (Sigma). Cells were washed twice with PBS and were gently mixed with AO/EB (1:100) dye solution for 1 minute; afterwards, the cells were observed and photographed under a Nikon Eclipse Ti fluorescence microscope (Nikon, Tokyo, Japan). Cells cultured without drug were considered as experimental control.

Databases. RUNX1T1 expression was assessed in two independent datasets: King Khalid University Hospital (KKUH) and GEO GSE21510. Data were imported into GeneSpring 13.0 (Agilent Technologies). Raw data were subsequently normalized using Percentile Shift, while the Benjamini-Hochberg false discovery rate method was used for multiple testing corrections. Two-fold cut-off and $p$ (corr) $<0.05$ were used to determine significantly changed transcripts. Expression of RUNX1T1 in different human cancer cell lines was interrogated using the Cancer Cell Line Encyclopedia (CCLE) database (https://portals.broadinstitute.org/ccle).

Statistical analysis. Statistical analyses and graphing were performed using Microsoft Excel 2010 (Redmond, Washington, USA) and GraphPad Prism 6.0 software (GraphPad, San Diego, CA, USA). $p$-Values were calculated using the two-tailed $t$-test.

\section{Results}

RUNX1T1 is down-regulated in primary CRC tissue and cell lines. Our previous mRNA expression profiling in CRC compared to adjacent normal tissues revealed multiple dysregulated mRNAs, including down-regulation of RUNXIT1 (2). The expression level of RUNX1T1 from our cohort of KKUH patients with CRC and from the GEO database both showing significant down-regulation of RUNXT1 in primary CRC compared to normal tissues (Figure 1a and b). Furthermore, the expression of RUNX1T1 in the CCLE database revealed CRC as the second most down-regulated cancer type (Figure 1c). Taken together, these data suggest a plausible role for RUNX1T1 in CRC.

Overexpression of RUNX1T1 suppresses CRC cells by regulating multiple cellular processes. In order to investigate the role of RUNX1T1 in CRC, we employed a lentiviral system to overexpress RUNX1T1 in the HCT116 CRC cell line. Stable expression of RUNX1T1 reduced the proliferation of HCT116 cells in vitro (Figure 2a). We subsequently performed global gene-expression profiling comparing RUNX1T1-transfected and control HCT116 cells, which revealed significant changes in gene expression between the two cell lines (Figure 2b). The top 10 altered pathways in RUNX1T1-transduced vs. control cells revealed an important role for RUNX1T1 in regulating the cell cycle, integrated cancer pathway, DNA replication and synthesis (Figure 2c).

Forced expression of RUNX1T1 increases sensitivity of CRC cells to 5-FU. Our pathway analysis of down-regulated genes in RUNX1T1-transduced HCT116 cells revealed response to DNA damage among the top altered pathways, suggesting that overexpression of RUNX1T1 might sensitize cancer cells to DNA damage-inducing agents (Figure 2c). Illustration of the DNA damage-response pathway is shown in Figure 3a, with the matched entities in RUNX1T1-transduced cells indicated.

In order to validate if $R U N X 1 T 1$ is indeed involved in regulating the response of CRC to DNA damage-inducing agents, we used the AO/EB assay to measure cell death in lentiviral control and RUNX1T1-transduced HCT116 cells in the presence of 5-FU. 5-FU at $>3.125 \mu \mathrm{M}$ was toxic to both cell types; however, using a lower concentration was more toxic and induced more cell death of RUNX1T1- 

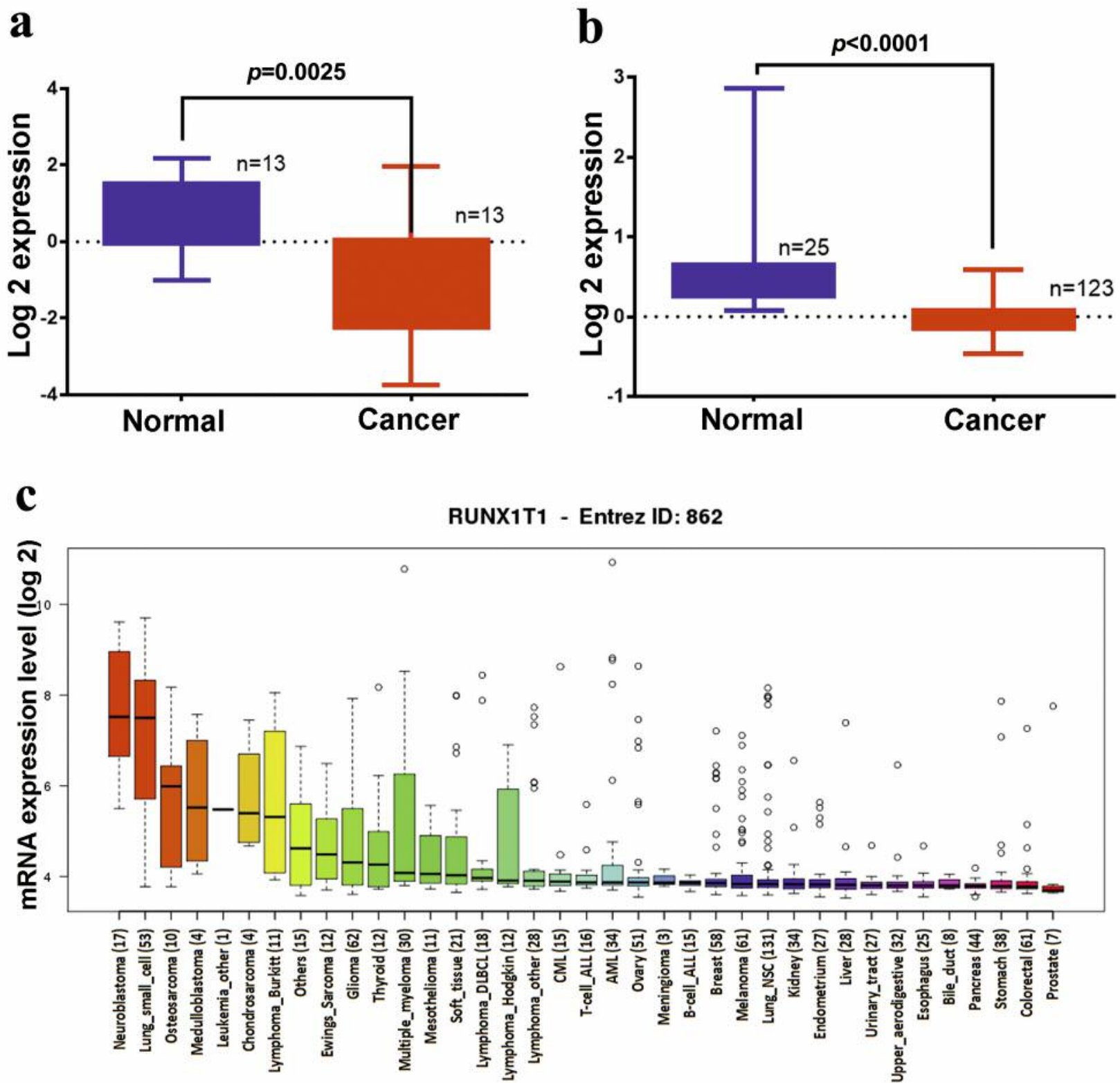

Figure 1. Runt-related transcription factor 1 (RUNX1T1) is down-regulated in primary colorectal cancer tissue and cell lines. Expression of RUNX1T1 in two colorectal cancer cohorts King Khalid University Hospital (KKUH) (a) and GEO GSE21510 (b) both showing significant downregulation of RUNXT1 in primary colorectal cancer compared to normal tissue. Data are presented using box and whiskers plots, whereby whiskers indicate minimum to maximum values and the box extends from the 25th to 75th percentiles. $c$ : Expression of RUNXIT1 across different human cancer cell lines showing severe down-regulation of RUNX1T1 in colorectal cancer cell lines. Expression levels of RUNXIT1 in cell lines were retrieved from the Cancer Cell Line Encyclopedia database. The numbers in parenthesis indicate the number of cancer cell lines in each particular cancer type.

transduced HCT116 compared to control HCT116 cells on day 6 (Figure 3b). A schema depicting a role for RUNX1T1 in CRC through regulation of the cell cycle, DNA replication, DNA damage, and estrogen signaling is shown in Figure 4.

\section{Discussion}

Mutations trigger proto-oncogenes through structural modifications in their encoded proteins. These modifications, which commonly involve vital protein regulatory domains, 
$\mathbf{a}$

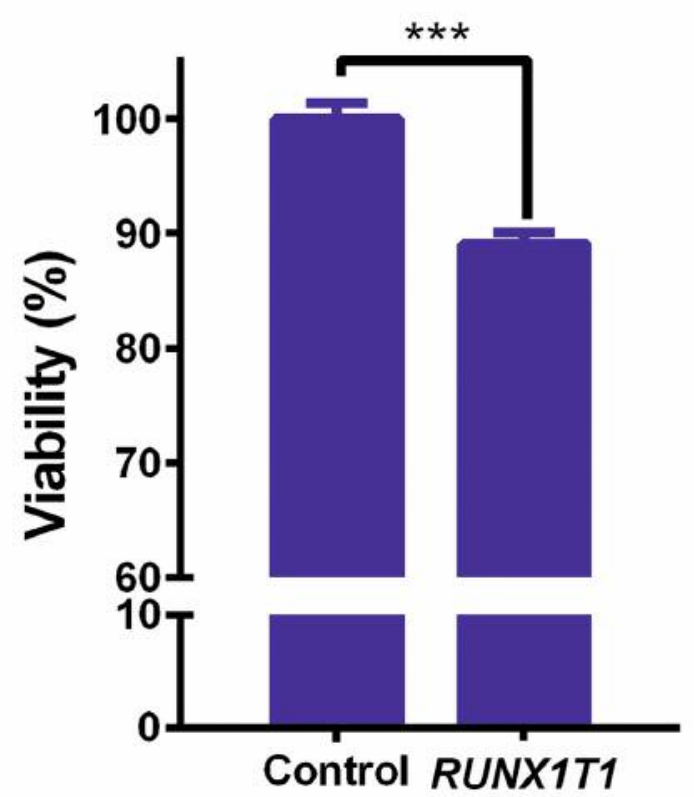

b

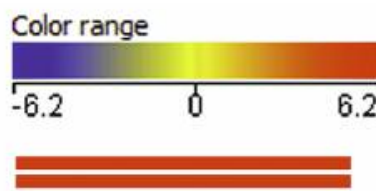

\section{Control}

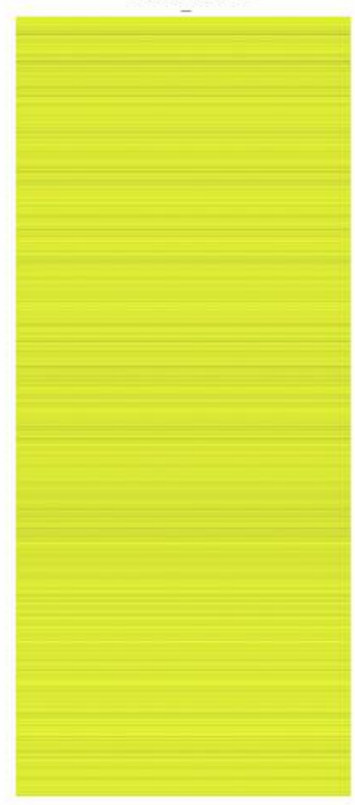

\section{RUNX1T1}

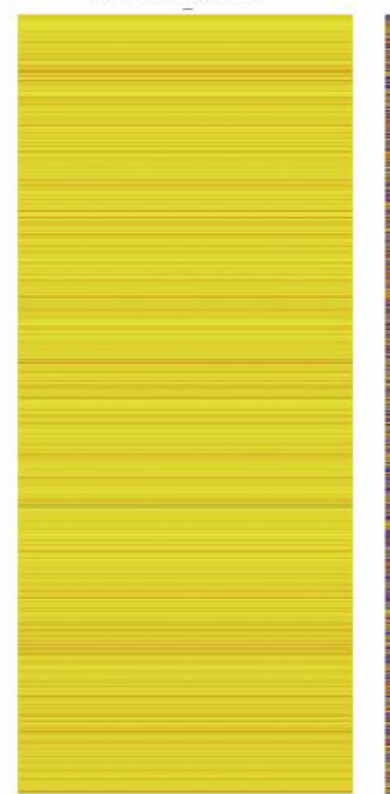

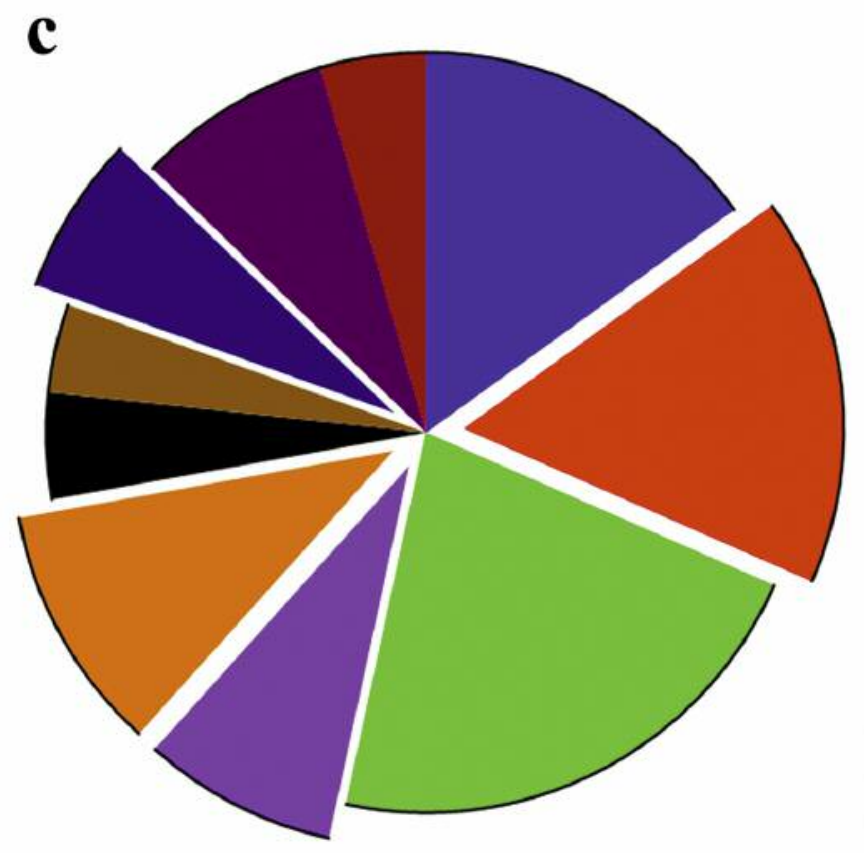

Hs_G1_to_S_cell_cycle_control_WP45_41128

$\square$ Hs_Cell_cycle_WP179_45137

$\square$ Hs_Integrated_Breast_Cancer_Pathway_WP1984_44857

$\square$ Hs_DNA_Replication_WP466_41036

$\square$ Hs_DNA_damage_response_WP707_38403

- Hs_Circadian_Clock_WP1797_42020

$\square$ Hs_Synthesis_of_DNA_WP1925_45107

- Hs_Integrated_Cancer_pathway_WP1971_44469

- Hs_Complement_and_Coagulation_Cascades_WP558_41101

$\square$ Hs_Estrogen_signaling_pathway_WP712_34842

Figure 2. Runt-related transcription factor 1 (RUNXIT1) suppresses colorectal cancer cells by regulating multiple cellular processes. a: Lentiviralmediated stable expression of RUNX1T1 reduces HCT116 colorectal cancer cell proliferation in vitro. Data are presented as mean $\pm S . E ., n=17 . b$ : Heat map depicting the global changes in gene expression between RUNX1T1-transduced and control HCT116 cells. c: Pie chart illustrating the distribution of the top 10 pathway designations for the differentially expressed genes in RUNX1T1-transduced compared to control HCT116 cells. The pie chart size corresponds to the number of matched entities. $* * * p<0.0005$. 

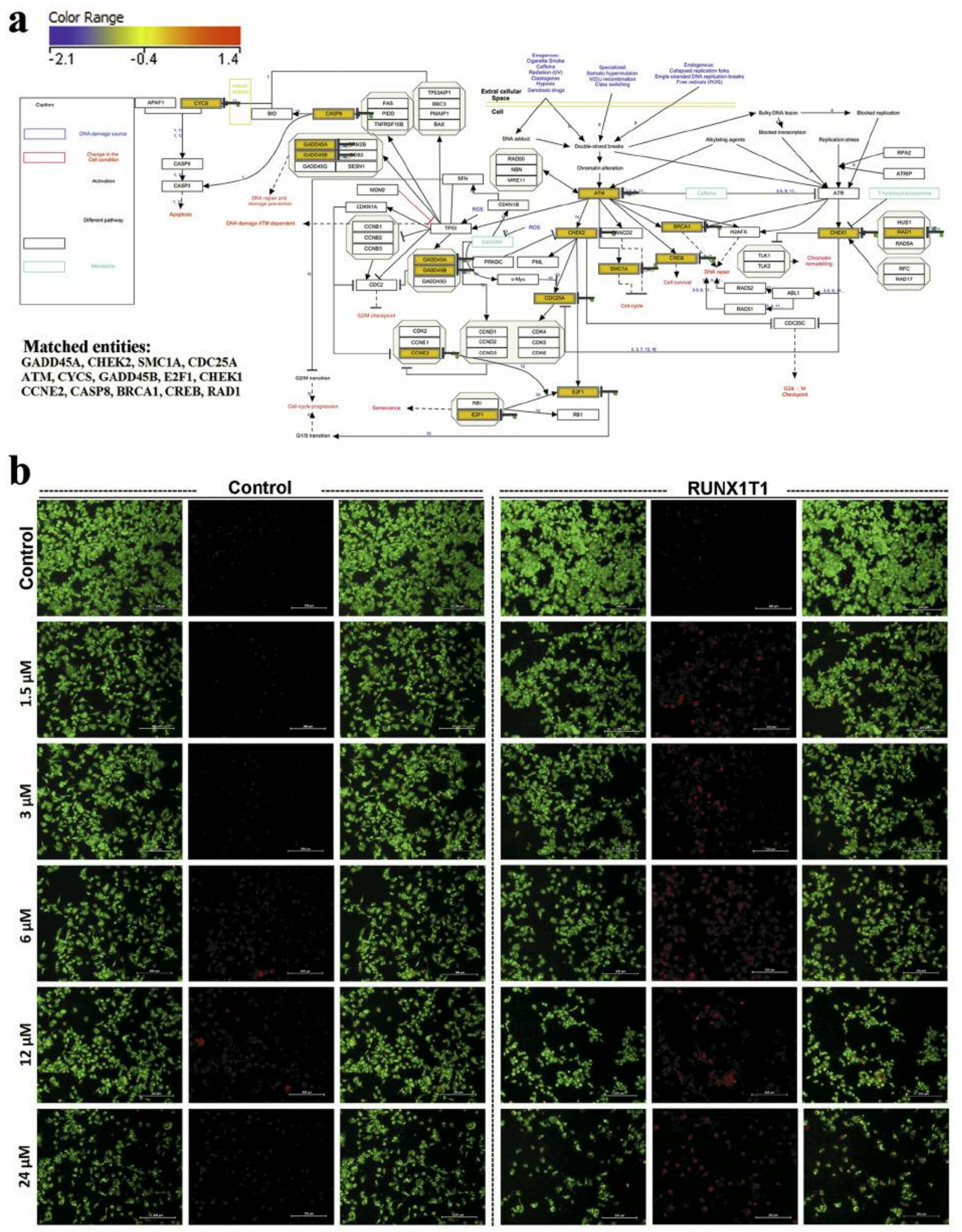

Figure 3. Role of runt-related transcription factor 1 (RUNX1T1) in promoting colorectal cancer. a: Illustration depicting the DNA damage-response pathway in RUNX1T1-transduced HCT116 cells with matched entities within the pathway indicated. $b$ : Representative fluorescence images of RUNX1T1-transduced and control HCT116 cells with/without different concentrations (1.5-24 $\mu \mathrm{M})$ of 5-fluorouracil. Cells were stained with acridine orange/ethidium bromide in order to detect apoptotic and necrotic cells. 

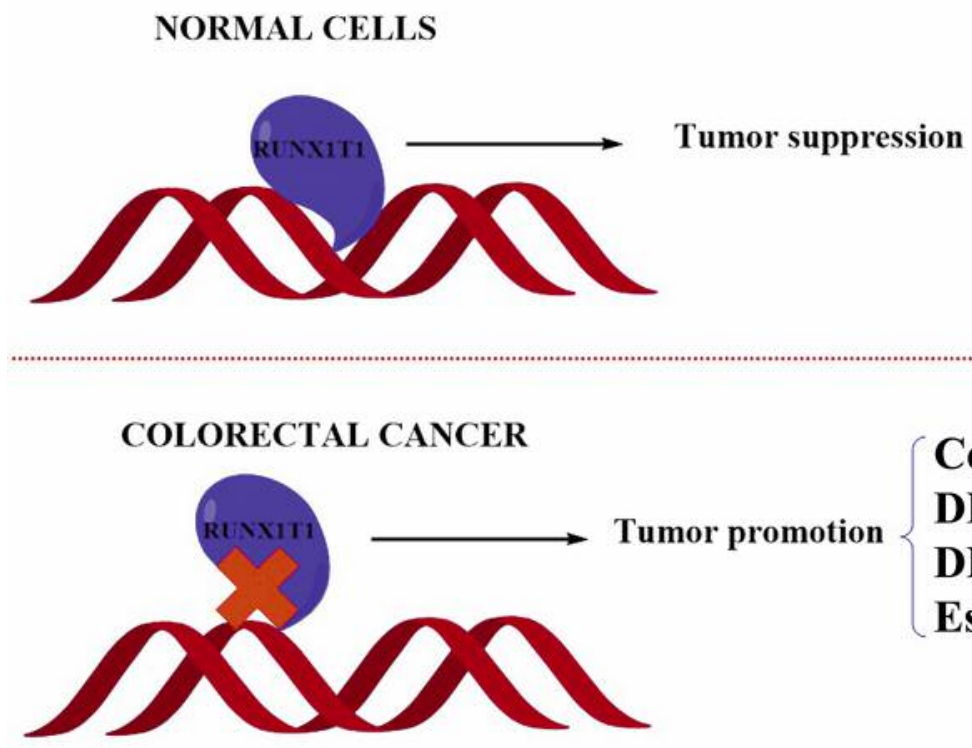

\section{Cell cycle regulation DNA damage response DNA replication Estrogen signaling}

Figure 4. Runt-related transcription factor 1 (RUNX1T1) regulates colorectal cancer (CRC) via multiple mechanisms. Schema depicting the role for RUNX1T1 in promoting colorectal cancer where in normal cells, RUNX1T1 is expressed, therefore several proto-oncogenes are suppressed (upper panel). In cancer cells, RUNX1T1 expression is diminished, therefore a number of genes in cell-cycle regulation, DNA replication, DNA damage response, or estrogen receptor signaling are expressed, that lead to colorectal cancer.

could potentially lead to the constitutive activation or inactivation of protein function (7). RUNX proteins are a family of evolutionarily conserved heterodimeric transcription factors that serve as master regulators of development. They are frequently deregulated in human diseases, including cancer, indicating a prominent and at times unpredictable role in cancer pathogenesis (11). The circumstantial signals that direct RUNX represent a fastgrowing arena in cancer research and could offer insights that are applicable to primary cancer diagnosis and treatment. Phenotypic effects of RUNX gene aberrations in human diseases have been reviewed by Ito $\mathrm{Y}$ et al., mainly $R U N X 1, R U N X 2$, and $R U N X 3$ (11). Among these, RUNX2 in stomach cancer, RUNX3 in colonic cancer were associated with loss of heterozygosity, hypermethylation and cytoplasmic localization (12).

Among the RUNX family, RUNX1T1 acted as an oncogene or as a tumor-suppressor gene (13-15). However, few studies have revealed the existence of RUNX1T1 mutations in various solid tumors, including CRC $(6,8)$, hence the role of RUNX1T1 in tumor progression, particularly CRC, is currently not well understood. Our previous work on CRC revealed multiple mRNA, miRNA and miRNA-mRNA regulatory networks, and proposed the increase or decrease of several mRNAs as a key mechanism leading to CRC development and progression. Interestingly, we found RUNX1T1 to be down-regulated in CRC, which was subsequently confirmed in a second cohort of patients with CRC from the GEO database. Concordantly, the CCLE database also showed the down-regulation of RUNX1T1, particularly in CRC (ranked as the second most downregulated) compared to 36 other cancer types. RUNX1T1 has been suggested as a novel biomarker for the prediction of liver metastasis, since the protein was underexpressed in well-differentiated metastatic primary pancreatic endocrine tumors compare to non-metastatic primaries (13).

Herein, we found the overexpression of RUNX1T1 to reduce the proliferation of HCT116 CRC cells. Pathway analyses revealed the cell cycle, integrated cancer pathway, DNA replication and synthesis, and DNA damage to be among the pathways most affected by RUNX1T1. Chemosensitivity was previously assessed in normal human cells (expressing RUNX1-RUNX1T1 as a single abnormality) with various drugs commonly used to treat acute myeloid leukemia. Abnormally, none of these agents differentially affected the growth of RUNX1-RUNX1T1-transduced cells, whereas, the combination of two or more of these chemotherapeutic agents reduced the concentration required to achieve $50 \%$ reduction in cell growth (15). In our study, RUNX1T1 increased the sensitivity of CRC cells to 5-FU. Our pathway analysis of genes down-regulated in RUNX1T1-transduced HCT116 cells revealed DNA-damage reponse among the most affected pathway, suggesting that overexpression of RUNX1T1 might sensitize cancer cells to DNA damage-inducing agents. When exposed to 5-FU, concentrations $>3.125 \mu \mathrm{M}$ were toxic to both control and RUNX1T1-transduced cells. Interestingly, 
lower concentrations were more toxic and induced more cell death in RUNX1T1-transduced compared to control HCT116 cells on day 6 . Therefore, overall, we observed overexpression of RUNX1T1 combined with 5-FU reduced proliferation and viability of CRC cells. The combination of 5-FU and other chemotherapeutic agents might exert more potent toxic effects on RUNX1T1-transduced cells.

In conclusion, based on our newly generated data, we would like to propose a model (see Figure 4), whereby loss of $R U N X 1 T 1$ expression results in CRC progression through pathways affecting the cell cycle, DNA damage, DNA replication, estrogen signaling, and drug resistance. Therefore, we would like to propose that restoring the expression of RUNX1T1 might serve as a potential therapeutic strategy in the future management of CRC.

\section{Acknowledgements}

This project was funded by the National Plan for Science, Technology and Innovation (MAARIFAH), King Abdulaziz City for Science and technology, Kingdom of Saudi Arabia, award number (11-MED-1942-02). We would like to thank Ms Rimi Hamam for her assistance with the microarray experiment.

\section{References}

1 Alajez NM: Significance of BMI1 and FSCN1 expression in colorectal cancer. Saudi J Gastroenterol 22: 288-293, 2016.

2 Vishnubalaji R, Hamam R, Abdulla MH, Mohammed MA, Kassem M, Al-Obeed O, Aldahmash A and Alajez NM: Genome-wide mRNA and miRNA expression profiling reveal multiple regulatory networks in colorectal cancer. Cell Death Dis 6: e1614, 2015.

3 Migas AA, Mishkova OA, Ramanouskaya TV, Ilyushonak IM, Aleinikova OV, and Grinev VV: RUNX1T1/MTG8/ETO gene expression status in human $\mathrm{t}(8 ; 21)(\mathrm{q} 22 ; \mathrm{q} 22)$-positive acute myeloid leukemia cells. Leuk Res 38: 1102-1110, 2014.

4 Dunne J, Cullmann C, Ritter M, Soria NM, Drescher B, Debernardi S, Skoulakis S, Hartmann O, Krause M, Krauter J, Neubauer A, Young BD and Heidenreich O: siRNA-mediated AML1/MTG8 depletion affects differentiation and proliferationassociated gene expression in $\mathrm{t}(8 ; 21)$-positive cell lines and primary AML blasts. Oncogene 25: 6067-6078, 2006.

5 Erickson P, Gao J, Chang KS, Look T, Whisenant E, Raimondi S, Lasher R, Trujillo J, Rowley J and Drabkin H: Identification of breakpoints in $\mathrm{t}(8 ; 21)$ acute myelogenous leukemia and isolation of a fusion transcript, AML1/ETO, with similarity to Drosophila segmentation gene, runt. Blood 80: 1825-1831, 1992.
6 Kan Z, Jaiswal BS, Stinson J, Janakiraman V, Bhatt D, Stern HM, Yue P, Haverty PM, Bourgon R, Zheng J, Moorhead M, Chaudhuri S, Tomsho LP, Peters BA, Pujara K, Cordes S, Davis DP, Carlton VE, Yuan W, Li L, Wang W, Eigenbrot C, Kaminker JS, Eberhard DA, Waring P, Schuster SC, Modrusan Z, Zhang Z, Stokoe D, de Sauvage FJ, Faham M and Seshagiri S: Diverse somatic mutation patterns and pathway alterations in human cancers. Nature 466: 869-873, 2010.

7 Kim YR, Kim MS, Lee SH and Yoo NJ: Mutational analysis of RUNX1T1 gene in acute leukemias, breast and lung carcinomas. Leuk Res 35: e157-158, 2011.

8 Wood LD, Parsons DW, Jones S, Lin J, Sjoblom T, Leary RJ, Shen D, Boca SM, Barber T, Ptak J, Silliman N, Szabo S, Dezso Z, Ustyanksky V, Nikolskaya T, Nikolsky Y, Karchin R, Wilson PA, Kaminker JS, Zhang Z, Croshaw R, Willis J, Dawson D, Shipitsin M, Willson JK, Sukumar S, Polyak K, Park BH, Pethiyagoda CL, Pant PV, Ballinger DG, Sparks AB, Hartigan J, Smith DR, Suh E, Papadopoulos N, Buckhaults P, Markowitz SD, Parmigiani G, Kinzler KW, Velculescu VE and Vogelstein B: The genomic landscapes of human breast and colorectal cancers. Science 318: 1108-1113, 2007.

9 Hamam D, Ali D, Vishnubalaji R, Hamam R, Al-Nbaheen M, Chen L, Kassem M, Aldahmash A and Alajez NM: microRNA320/RUNX2 axis regulates adipocytic differentiation of human mesenchymal (skeletal) stem cells. Cell Death Dis 5: e1499, 2014.

10 Vishnubalaji R, Hamam R, Yue S, Al-Obeed O, Kassem M, Liu FF, Aldahmash A and Alajez NM: MicroRNA-320 suppresses colorectal cancer by targeting SOX4, FOXM1, and FOXQ1. Oncotarget, 2016. doi: 10.18632/oncotarget.8937. [Epub ahead of print]

11 Ito Y, Bae SC and Chuang LS: The RUNX family: developmental regulators in cancer. Nat Rev Cancer 15: 81-95, 2015.

12 Chuang LS and Ito Y: RUNX3 is multifunctional in carcinogenesis of multiple solid tumors. Oncogene 29: 26052615,2010

13 Nasir A, Helm J, Turner L, Chen DT, Strosberg J, Hafez N, Henderson-Jackson EB, Hodul P, Bui MM, Nasir NA, Hakam A, Malafa MP, Yeatman TJ, Coppola D and Kvols LK: RUNX1T1: a novel predictor of liver metastasis in primary pancreatic endocrine neoplasms. Pancreas 40: 627-633, 2011.

14 Tonks A, Pearn L, Musson M, Gilkes A, Mills KI, Burnett AK and Darley RL: Transcriptional dysregulation mediated by RUNX1-RUNX1T1 in normal human progenitor cells and in acute myeloid leukaemia. Leukemia 21: 2495-2505, 2007.

15 Tonks A, Pearn L, Mills KI, Burnett AK and Darley RL: The sensitivity of human cells expressing RUNX1-RUNX1T1 to chemotherapeutic agents. Leukemia 20: 1883-1885, 2006.

Received August 22, 2016

Revised September 15, 2016

Accepted September 22, 2016 